\title{
ANALYSIS OF PATIENTS WITH LOCALLY ADVANCED BREAST CANCER TREATED AT ICESP
}

Gabriela Bezerra Nobrega', Bruna Salani Mota1', Gabriela Boufelli de Freitas¹, Jonathan Yugo Maesaka1, Rodrigo Gonçalves', Sérgio Mitsuo Masili Oku¹, Angela Francisca Trinconi da Cunha', José Roberto Filassi ${ }^{1}$

${ }^{1}$ Setor de Mastologia, Faculdade de Medicina, Universidade de São Paulo - São Paulo (SP), Brazil.

Objective: The aim of this study was to assess the oncological efficacy of breast-conserving surgery (BCS) after neoadjuvant therapy (NT) in patients with invasive locally advanced breast cancer (LABC). Methodology: A retrospective cohort study was conducted at the Instituto do Cancer de São Paulo Octavio Frias de Oliveira (ICESP), with LABC (Stages IIbIII) treated with NT between 2010 and 2015. The endpoints were disease-free survival (DFS), local disease-free survival (LDFS), overall survival (OS), and residual tumor volume, considering pathological complete response (PCR) as ypT0 ypN0. The multivariable analyses were performed by using the Cox proportional hazard models. Results: In this study, 529 patients were included. The mean age was 52.7 (51.53-53.90). All patients were submitted to NT, i.e., $95.5 \%$ was submitted to neoadjuvant chemotherapy and 4.5\%, hormone therapy. The mean follow-up was 62.33 (60.01-64.65) months. The PCR was identified in $12.7 \%$. The BCS was performed in $24.6 \%$ (130) patients versus $75.4 \%$ (399) of mastectomies (MTs). There were no differences in $13 \%$ versus 9.2\% (95\%CI; $\mathrm{p}=0.2$ ) LDFS for MT and BCS. The DFS was lower at $55.4 \%$ in the MT group versus $77.7 \%$ in the BCS group $(95 \% \mathrm{CI} ; \mathrm{p}<0.001)$. The mortality rate was $29.5 \%$. In multivariable, the following factors were associated with higher risk of mortality: non-PCR (relative risk $[R R] 2.23$; 95\%CI 1.173-4.242; $p=0.002$ ), pathological stage 3B or 3C (RR 2.193; 95\%CI 1.377-3.492; $\mathrm{p}=0.004$ ), and Ki67>30 (RR1.8; 95\%CI 1.331-2.618; $\mathrm{p}=0.000)$. The type of surgery had no impact on mortality (RR 1.47; 95\% CI 0.945-2.298; $\mathrm{p}=0.08$ ). Conclusion: In our population, the BCS does not affect the LDFS rates and mortality, which seems to be safe to perform in patients who desire to conserve the breast after neoadjuvant treatment. PCR, clinical stage, and Ki67 had an important impact on mortality.

Keywords: Locally Advanced Breast Cancer; Neoadjuvant Therapy; Pathological Complete Response. 